\title{
Charge-state-dependent collisional energy-loss straggling of swift ions in a degenerate electron gas
}

\author{
I. Nagy ${ }^{1,2}$ and I. Aldazabal ${ }^{3,2}$ \\ ${ }^{1}$ Department of Theoretical Physics, Institute of Physics, Technical University of Budapest, H-1521 Budapest, Hungary \\ ${ }^{2}$ Donostia International Physics Center (DIPC), P. Manuel de Lardizabal 4, 20018 San Sebastián, Spain \\ ${ }^{3}$ Centro de Física de Materiales (CSIC-UPV/EHU)-MPC, P. Manuel de Lardizabal 3, 20018 San Sebastián, Spain
}

(Received 23 September 2009; published 14 December 2009)

\begin{abstract}
In order to characterize the statistical aspect of the energy loss in particle penetration, Bohr developed a kinetic theory and applied it to a beam of fast $\alpha$ particles interacting with free electrons. The present study rests on this classical theory of collisional straggling, and it is implemented by using a partially screened Coulomb potential to model the electron-projectile interaction. The deflection angle of electron scattering in this longranged field is calculated analytically within the framework of classical mechanics. The transport fluctuation cross section, which is the basic quantity to the collisional straggling in Bohr's modeling, is determined numerically. By varying the number of bound electrons around the swift He ions, the effect of prefixed charge states in the collisional energy-loss straggling is quantified. An incoherent weighted summation of different fixed charge-state channels is discussed as well, by using normalized probabilities.
\end{abstract}

DOI: 10.1103/PhysRevA.80.064901

PACS number(s): $34.50 . \mathrm{Bw}$

The statistical nature of the energy dissipation of heavy projectiles interacting with different targets requires the consideration of the energy-loss straggling which characterizes the broadening of the beam in the penetration phenomenon, i.e., it describes [1] the fluctuation in the energy-loss process. Apart from its intrinsic theoretical interest, the straggling is an important quantity in ion-beam based techniques for material structuring, and in hadron therapy also. Clearly, the quantitative role of different charge-states is a fundamental issue [2-8] in a proper description of the energy-loss phenomenon. The present theoretical study on the collisional energy-loss straggling is devoted to this problem.

Based on Bohr's classical theory, which seems to constitute one of the most lasting results of the theory of particle penetration, the mean square fluctuation in the energy loss $\left[\Omega^{2}=\left\langle(\Delta E-\langle\Delta E\rangle)^{2}\right\rangle\right.$ where $\Delta E$ is the energy loss in a twobody collision] is as follows:

$$
\Omega^{2}=\left(\frac{T_{0}}{2}\right)^{2} \sigma_{t r f}(p)\left(n_{0} \Delta x\right)
$$

for an electron gas target [9]. Here $\Delta x$ is the penetration length, $p=m v$ the momentum of electrons in relative motion, and $n_{0}$ the density of the electron gas. The maximal energy transfer is $T_{0}=m_{e}(2 v)^{2} / 2$ in an elastic two-body collision between the swift heavy projectile moving with velocity $v$ and an electron. Atomic units, $\hbar=m_{e}=e^{2}=1$, are used below.

The transport fluctuation (trf) cross section to Eq. (1) is defined as follows:

$$
\sigma_{t r f}(p)=\int d \sigma(1-\cos \vartheta)^{2},
$$

in terms of the differential cross section $(d \sigma)$ and the scattering angle $(\vartheta)$. This integrated cross section contains those details which are determined by the potential form in an elastic two-body collision. Bohr's theory rests on the series of such, statistically independent events. Notice that, since $(1-\cos \vartheta)^{2}=4 \sin ^{4}(\vartheta / 2)$, the straggling depends on the square of $\left(\Delta E / T_{0}\right) \sim \sin ^{2}(\vartheta / 2)$ which is prescribed by standard conservation laws.

Recently, the above cross section was determined [10] within the framework of classical mechanics for a wellknown $[11,12]$ finite-range attractive $(Z>0)$ potential form

$$
V_{s}(r)=-\frac{Z}{r}\left(1-\frac{r}{R_{S}}\right),
$$

with the $V_{s}(r)=0$ constraint for $r \geq R_{s}$. The analytic result obtained is the following

$$
\sigma_{t r f}^{s}(v)=\pi R_{s}^{2} \frac{4}{(A-1)^{2}}\left[(A+1)-\left(\frac{2 A}{A-1}\right) \ln A\right],
$$

where $A=\left[v^{2}\left(R_{s} / Z\right)-1\right]^{2}$ is a convenient shorthand. The Coulombic case, treated by Bohr in his pioneering work on straggling, corresponds to the $R_{S} \rightarrow \infty$ mathematical limit. It was pointed out [10] that the ratio $\left[\sigma_{t r f}^{s}\left(R_{s}, v\right) / \sigma_{t r f}^{s}\left(R_{s}=\infty, v\right)\right]$ could reach a maximal value of $4 / 3$ under the $\left[\left(Z / R_{s}\right) / v^{2}\right]=1 / 2$ condition which is, in fact, also the condition [12] behind the so-called giant Coulomb glory effect [see the discussion at Eq. (9) below].

As the most natural extension of the previous results, which also allows a physical transition to the Coulombic limit treated by Bohr, we shall use a partially screened form

$$
V_{p s}(r)=-\frac{(Z-N)}{r}-\frac{N}{r}\left(1-\frac{r}{R_{p s}}\right),
$$

for $r<R_{p s}$, and take $V_{p s}(r)=(Z-N) / r$ for $r \geq R_{p s}$. The parameter $R_{p s}$ will be specified below. With $N=Z$, we get back a finite-range $\left(R_{s}=R_{p s}\right)$ potential discussed above. Note that the bare Coulomb case is defined (when $Z$ and $N$ are fixed) by the $R_{p s} \rightarrow \infty$ limit, at which we have the $V(r)=-Z / r$ form applied $[1,9]$ earlier to the collisional energy-loss straggling. In the present work the different channels, which are due to electron capture and loss processes, are modeled via the $N$ and $R_{p s}(N)$ input parameters. 
In the determination of the scattering angle based on classical mechanics with our potential in Eq. (5), there are impact parameters $(b)$ with $b \geq b_{*}$ where only the $V_{p s}(r)=-(Z-N) / r$ potential term govern the trajectory contributing to the final rotation angle. This important $b_{*}$ value is determined from the $r_{\min } \geq R_{p s}$ constraint, where $r_{\min }$ is the (b-dependent) closest approach in our potential. From this constraint one gets

$$
b_{*}=R_{p s}\left[1+2 \frac{(Z-N)}{R_{p s} v^{2}}\right]^{1 / 2} .
$$

This informative expression shows the role of interplay between kinetic and potential energies. After a straightforward but long algebra, the impact-parameter-dependent scattering angle $[\vartheta(b)]$ becomes

$$
\begin{aligned}
\left(\frac{\vartheta}{2}\right)_{p s}= & \arctan \left(\frac{Z-N}{b v^{2}}\right)+\Theta\left(b_{*}-b\right) \arctan \\
& \times\left[\frac{N}{R_{p s}} \frac{b \sqrt{b_{*}^{2}-b^{2}}}{b^{2}\left(v^{2}-N / R_{p s}\right)+Z(Z-N) / v^{2}}\right],
\end{aligned}
$$

where $\Theta$ is the Heaviside (generalized) function.

In the completely screened case $\left(N=Z\right.$ with $R_{p s}=R_{s}$, thus $\left.b_{*}=R_{s}\right)$ we have

$$
\left(\frac{\vartheta}{2}\right)_{s}=\arctan \left[\frac{Z}{b} \frac{\sqrt{1-b^{2} / R_{s}^{2}}}{\left(v^{2}-Z / R_{s}\right)}\right],
$$

for the allowed $b \in\left[0, R_{s}\right]$ values $[13,14]$. Finally, the Coulomb case is defined, as we mentioned above, by the $R_{p s} \rightarrow \infty$ limit in Eq. (7) with Eq. (6), which results in

$$
\left(\frac{\vartheta}{2}\right)_{C}=\arctan \left(\frac{Z}{b v^{2}}\right) \text {. }
$$

The strongest restriction to get single-valued $\vartheta(b)$ functions from Eqs. (7) and (8) is $\left(R_{s} v^{2} / Z\right) \geq 1$. This constraint is in a perfect harmony with a recent statement [12]. When it is satisfied, one has only one classical trajectory which contributes to the scattering at a given $\vartheta$.

It is very useful to discuss the above expressions at small values of the impact parameter. A two-term Taylor expansion gives the $\vartheta \simeq \pi-\left(2 b v^{2} / Z\right)$ limit for the Coulomb case. The expansion of Eq. (8) results in $\vartheta \simeq \pi-\left(2 b v^{2} / Z\right)\left[1-Z /\left(R_{s} v^{2}\right)\right]$, and one gets from Eq. (7) with $N \neq Z$ the $\vartheta \simeq \pi-\left(2 b v^{2} / Z\right)[Z /(Z-N)]\left[1-N b_{*} /\left(Z R_{p s}\right)\right]$ form. Clearly, the effect of screening (complete or partial) extends the domain of effective backscattering, i.e., at small impact parameters the scattering angle is larger when there is screening. This is the phenomenological explanation behind the above-mentioned Coulomb glory effect.

We note at this point that the special dependence of the integrand function in Eq. (2) on the scattering angle makes our description with a classical differential cross section a well-balanced one. It is well known [15] that the classical scattering angle decreases faster at sufficiently large impact parameters than does the wave-mechanical diffraction angle. The special weighting removes, to a large extent, this range of the $b$ variable. In addition, by decreasing the screening one approaches the Coulombic values for the scattering angle $\vartheta(b)$, but in an interesting manner. Namely, from above at small impact parameters, and from below $(N \neq Z)$ at large impact parameters. This remarkable behavior in the screened case, modulated by its intrinsic velocity dependence, makes the deviation from Bohr's straggling a challenging theoretical problem. Qualitatively, there is a nontrivial interplay between a decreasing glory effect and a growing impact-parameter range in integration.

Using Eqs. (7)-(9), and standard addition and transformation rules in trigonometry, one can easily derive the important $\sin ^{4}(\vartheta / 2)$ weighting factor to Eq. (2) in terms of the input parameters $\left\{Z, N, v, R_{p s}\right\}$, and the $b$ variable. For the $b \in\left[0, b_{*}\right]$ range we derive

$\sin ^{4}\left(\frac{\vartheta}{2}\right)_{p s}=\frac{\left[(Z-N)\left(b^{2}+K_{2}\right) / v^{2}+b^{2} K_{1} \sqrt{b_{*}^{2}-b^{2}}\right]^{4}}{\left[b^{2}+(Z-N)^{2} / v^{4}\right]^{2}\left[\left(b^{2}+K_{2}\right)^{2}+b^{2} K_{1}^{2}\left(b_{*}^{2}-b^{2}\right)\right]^{2}}$,

where $K_{1}=\left(N / R_{p s}\right) /\left[v^{2}-\left(N / R_{p s}\right)\right]$ and $K_{2}=\left[Z(Z-N) / v^{2}\right] /$ $\left[v^{2}-\left(N / R_{p s}\right)\right]$. For the so-called Coulombic part, $b \in\left[b_{*}, \infty\right)$, the result is more simple

$$
\sin ^{4}\left(\frac{\vartheta}{2}\right)=\left(\frac{Z-N}{v^{2}}\right)^{4} \frac{1}{\left[b^{2}+(Z-N)^{2} / v^{4}\right]^{2}} .
$$

We stress the point (see, above) that in the present treatment the bare Coulomb limit is defined from Eq. (10) by taking $R_{p s} \rightarrow \infty$, which results in

$$
\sin ^{4}\left(\frac{\vartheta}{2}\right)_{C}=\left(\frac{Z}{v^{2}}\right)^{4} \frac{1}{\left[b^{2}+\left(Z / v^{2}\right)^{2}\right]^{2}} .
$$

Finally, in the completely screened $(s)$ case one gets from Eq. (10) the simple

$$
\sin ^{4}\left(\frac{\vartheta}{2}\right)_{s}=K_{1}^{4} \frac{\left(R_{s}^{2}-b^{2}\right)^{2}}{\left[b^{2}+K_{1}^{2}\left(R_{s}^{2}-b^{2}\right)\right]^{2}},
$$

expression, since $K_{2}=0$ and $b_{*}=R_{s}$ when $N=Z$.

After the above details with prefixed charge states for intruders, we turn to the question of weighting. Of course, only some statistical concept [2-5] for a weightedsummation of channel-terms obtained above (at integer $N$ values) for straggling can provide a reasonable approximation. On the other hand, an accurate treatment of higherorder effects, which cause the jumps between various charge states in a statistical energy-loss process, is a complicated issue. In order to quantify the equilibrium-charge state fractions we need an experimental input or a separate quantum treatment. Our weighting in this work rests on equilibrium charge-state fractions applied earlier [5] in stopping power calculations for He ions. In the rest of the paper this particular case of projectiles will be considered.

The practical implementation of our classical theory requires an estimation for the screening parameter $R_{p s}$. We will use for it the radius at which the radial density of a $1 s$ state around the $\alpha$ particle has its maximum; $R_{p s}=1 / 2$. With $d \sigma$ $=2 \pi b d b$ in Eq. (2), we performed the integration for the partially screened $(N \neq Z)$ cases numerically. In the $R_{s} \rightarrow \infty$ limit we recover from Eq. (4) via expansion [or, at $R_{p s} \rightarrow \infty$, 


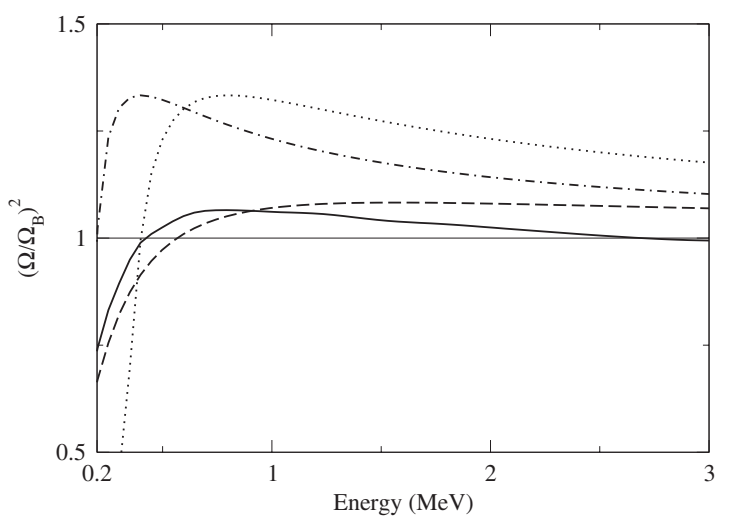

FIG. 1. Different straggling ratios, $\left(\Omega / \Omega_{B}\right)^{2}$, as a function of projectile energy. The dotted and dash-dotted curves are based on a completely screened $(Z=N=2)$ two-body interaction energy, i.e., on Eq. (3) with $R_{s}=1 / 2$ and $R_{S}=1$, respectively. The long-dashed curve rests on Eq. (5) for a partially screened $(Z=2, N=1)$ potential, with $R_{p s}=1 / 2$ to model an $\mathrm{He}^{+}$ion. The solid curve is calculated via Eq. (14). It describes a weighted average of channel contributions.

from our numerics which rests on Eqs. (10) and (11) to the definition in Eq. (2)] the well-known simple form $\sigma_{\text {trf }}^{C}(v)=4 \pi Z^{2} / v^{4}$ based on the bare Coulomb potential [1,9]. The corresponding energy-loss straggling, Bohr's $\Omega_{B}^{2}$, is used below as a natural measure to discuss deviations from it.

In Fig. 1, different ratios $\left[\Omega^{2} / \Omega_{B}^{2}\right]=\left[\sigma_{t r f}(v) / \sigma_{t r f}^{C}(v)\right]$ are plotted as a function of projectile-energy given in $\mathrm{MeV}$ units. The conversion to ion velocity in atomic units (a.u.) is given by $E(\mathrm{MeV})=0.1[v(\text { a.u. })]^{2}$ for the case $(Z=2)$ investigated in the present work. The dotted and dash-dotted curves are based on the potential-form given by Eq. (3), i.e., on a completely screened two-body potential energy. They are calculated via Eq. (4) for the $E \in[0.2,3] \mathrm{MeV}$ ion-energy range, with $R_{s}=1 / 2$ and $R_{s}=1$, respectively. The value of $R_{s}=1 / 2$ refers to a neutral-atom-unscreened-electron picture.

The other curves are based on the potential-form given by Eq. (5), i.e., on a partially screened two-body interaction energy. The long-dashed curve refers to the singly-ionized $\left(\mathrm{He}^{+}\right)$case. It is calculated under the prefixed $N=1$ and $R_{p s}=1 / 2$ conditions. Finally, the solid curve is based on an incoherent weighted-averaging $(a v)$ of results obtained (separately) for the different $\left(\mathrm{He}, \mathrm{He}^{+}\right.$, and $\left.\mathrm{He}^{2+}\right)$ chargestate channels

$$
\begin{aligned}
{\left[\frac{\Omega^{2}}{\Omega_{B}^{2}}\right]_{a v}=} & P\left(\mathrm{He}^{2+}\right)\left[\frac{\Omega^{2}}{\Omega_{B}^{2}}\right]_{\mathrm{He}^{2+}}+P\left(\mathrm{He}^{+}\right)\left[\frac{\Omega^{2}}{\Omega_{B}^{2}}\right]_{\mathrm{He}^{+}} \\
& +P(\mathrm{He})\left[\frac{\Omega^{2}}{\Omega_{B}^{2}}\right]_{\mathrm{He}},
\end{aligned}
$$

where $P\left(\mathrm{He}^{2+}\right), P\left(\mathrm{He}^{+}\right)$, and $P(\mathrm{He})$ are [5] normalized probabilities for the corresponding charge states, and they satisfy the $P\left(\mathrm{He}^{2+}\right)+P\left(\mathrm{He}^{+}\right)+P(\mathrm{He})=1$ constraint. Clearly, in our incoherent modeling the first product on the right-hand-side is just $P\left(\mathrm{He}^{2+}\right)$.

The curves calculated with a completely screened potential energy for the two-body interaction, show maxima whose positions depend [see the discussion at Eq. (4)] on $R_{s}$. Of course, all these curves $(Z=N=2)$ tend [10] to the Bohr result at high velocities as

$$
\left(\frac{\Omega}{\Omega_{B}}\right)_{\mathrm{He}}^{2} \cong 1+2 \frac{Z}{R_{s} v^{2}} .
$$

In the singly-ionized $(N=1)$ case, which is one of the usual charge state of incoming projectiles, the straggling is still higher than the Bohr result in an appreciable range of energies; asymptotically we have $\left(\Omega / \Omega_{B}\right)_{\mathrm{He}^{+}}^{2} \cong 1+2\left[N /\left(R_{p s} v^{2}\right)\right]$. Finally, the solid curve suggests that after an incoherent weighting of allowed charge states, the averaged straggling differs from Bohr's prediction with bare $\alpha$ particles only a little over a surprisingly broad energy range. This statement is important from a general point of view on the energy-loss phenomenon, since it is this energy range in which Rutherford performed his famous experiments with $\alpha$ particles and established his atomic model of condensed matter.

At this point of presenting numerical results, we would like to mention an other possibility of an average characterization of straggling. Such a treatment would rest still on the screened form in Eq. (3) but with the $Z \rightarrow Z_{\text {eff }}$ and $R_{s} \rightarrow R_{s}\left(Z_{e f f}\right)$ parametrization. This could mimic, in a meanfield picture, the variation of an effective charge (and thus of the screening) with ion velocity. A way of fixing the parameters could be to apply a constraint via (solely) that contribution to the stopping power which is due to single-particle scattering in a dielectric medium, including [16] the socalled $Z_{1}^{3}$ Barkas term. The closed expression derived earlier [10] for the transport ( $t r)$ cross section

$$
\sigma_{t r}\left(Z, R_{s}, v\right)=\pi R_{s}^{2} \frac{2}{(A-1)^{2}}[A \ln A-(A-1)]
$$

could allow $[A$ is given at Eq. (4) $]$ a future extension along this line, since it contains (in a limiting case, where $\left.\left[\left(Z / R_{s}\right) / v^{2}\right] \ll 1\right)$ the mentioned (perturbative) $Z_{1}^{3}$ charge-sign effect.

In conclusion, the charge-state dependence of the collisional energy-loss straggling has been investigated in this work by implementing Bohr's classical method using a partially screened two-body interaction potential. We solved the classical scattering problem exactly for such an interaction, thus considerably extending the proper phenomenology beyond the classic work of Bohr. We quantified the nontrivial interplay between the Coulomb glory effect and the effective impact-parameter range by varying the velocity of the swift attractive ion. The statistical weighting of contributions, calculated for different charge-state channels, is briefly discussed. As a future direction, we propose to investigate in more detail the space-time aspects $[17,18]$ of an effective two-body interaction by combining quantum-statistical and semiclassical (mean-field) charge-polarization [19] pictures. The present result obtained for $\mathrm{He}^{+}$suggests [see the discussion at Eq. (15)] that a further (dynamical) screening may act to enhance the Barkas effect, in agreement with an earlier [20] forecast. 
The authors are thankful to A. Arnau, P. M. Echenique, and A. Howie for very valuable discussions on various aspects of the stopping phenomenon. One of us (I.N.) acknowledges the generous hospitality at the DIPC, where this work has been completed. Financial support by the Spanish MCyT (Grant No. FIS2007-066711-CO2-00) is gratefully acknowledged.
[1] N. Bohr, Mat. Fys. Medd. K. Dan. Vidensk. Selsk. 18, 1 (1948).

[2] F. Guinea, F. Flores, and P. M. Echenique, Phys. Rev. B 25, 6109 (1982).

[3] P. M. Echenique, I. Nagy, and A. Arnau, Int. J. Quantum Chem. 23, 521 (1989).

[4] A. Narmann, R. Monreal, P. M. Echenique, F. Flores, W. Heiland, and S. Schubert, Phys. Rev. Lett. 64, 1601 (1990).

[5] A. Arnau, M. Penalba, P. M. Echenique, and F. Flores, Nucl. Instrum. Methods Phys. Res. B 69, 102 (1992).

[6] P. Sigmund, Phys. Rev. A 56, 3781 (1997).

[7] A. F. Lifschitz and N. R. Arista, Phys. Rev. A 69, 012902 (2004).

[8] R. M. Papaléo, M. R. Silva, R. Leal, P. L. Grande, M. Roth, B. Schattat, and G. Schiwietz, Phys. Rev. Lett. 101, 167601 (2008).

[9] J. Lindhard and A. H. Sorensen, Phys. Rev. A 53, 2443 (1996).

[10] I. Nagy, R. Vincent, J. I. Juaristi, and P. M. Echenique, Phys.
Rev. A 78, 012902 (2008).

[11] N. F. Mott and H. S. W. Massey, The Theory of Atomic Collision (Clarendon, Oxford, 1965).

[12] Yu. N. Demkov and V. N. Ostrovsky, J. Phys. B 34, L595 (2001).

[13] N. R. Arista, P. L. Grande, and A. F. Lifschitz, Phys. Rev. A 70, 042902 (2004).

[14] I. Nagy, Nucl. Instrum. Methods Phys. Res. B 94, 377 (1994).

[15] A. B. Migdal, Qualitative Methods in Quantum Theory (Benjamin, London, 1977).

[16] J. M. Pitarke, R. H. Ritchie, P. M. Echenique, and E. Zaremba, Europhys. Lett. 24, 613 (1993).

[17] I. Nagy, Phys. Rev. A 65, 014901 (2001).

[18] J. Tao, X. Gao, G. Vignale, and I. V. Tokatly, Phys. Rev. Lett. 103, 086401 (2009).

[19] Z. L. Miskovic, S. G. Davison, F. O. Goodman, and W.-K. Liu, Phys. Rev. B 60, 14478 (1999).

[20] P. Sigmund and A. Schinner, Phys. Rev. Lett. 86, 1486 (2001). 